\title{
THE ROLE OF CONTROL PROCESSES IN REGULATING SELF- EFFICACY IN TASKS WITH HIGH PREDICTABILITY
}

The research is funded by the Grant of President of Russian Federation № MK-1382.2020.6 Allakhverdov M.V., Lvova O.V. (SPSU, Saint-Petersburg, Russia) m.allakhverdov@spbu.ru,olya.lvova@gmail.com

乙tipluјшgर्ume uर्u. 31.07.2021

9pupunuर्ume uर्u. 02.09.2021

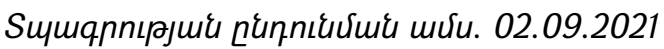

The study's objective is to describe how cognitive control mechanisms influence performance in different tasks. We believe that popular resource models have disadvantages when explaining one's effectiveness in a particular task. We argue that the limitations on one's performance happen not because of limited cognitive resources but rather due to logically based restrictions. One of the restrictions is rooted in one's need for consistency in their representations of the world and themselves. We infer that having a specific expectation about self-efficacy in a particular task dramatically influences the outcome: cognitive control mechanisms finetune one's performance to agree with one's expectations on the efficiency of said performance. In the study, we tested how regularities in stimuli influence one's performance. In the experiment (23 subjects), we used the Musical Stroop test. For part of the trials, we used melodies as regularities, while in other trials, we presented fillers - random sets of notes. The results show that melodies reduce interference and have a faster response time than in the filler condition. We argue that it is due to the role of cognitive control mechanisms that maintain self-efficacy. We discuss the implication of the proposed approach.

Keywords: self-efficacy; cognitive control; consistency; the Musical Stroop Test.

DOI: https://doi.org/10.46991/SBMP/2021.4.2.019

The effectiveness we demonstrate while performing a task mostly roots in our implicit representation of self-efficacy. If someone believes that they are successful in a particular activity, they will perform faster and more accurately than someone who thinks the opposite. This difference happens because our cognitive system strives for consistency. Consistency in our system of beliefs and concepts is essential because it allows a stable, predictable representation of the world and ourselves. Hence, we can plan our behaviour, have expectations, and make assumptions about the future - all of this helps us live our life. More than half a century ago, George Kelly noticed that inconsistency in one's representations leads to anxiety, frustration, or guilt [5]. Thus, we should consider maintaining consistency as the most important 
goal for our cognitive system. The cognitive system implements a set of processes that can influence our behaviour and the cognitive system itself for it to work. We can think about those processes as controlling mechanisms. Our previous findings and theoretical analysis allow us to assume the existence of at least three different controlling processes.

The first one is the Operational Control that checks whether one's behaviour matches the algorithm that the system believes is best suited for the task at hand. The second one is Task Control that verifies whether one's actions aim to check whether one solves the highest priority task (i.e., we can imagine this process as the system asking itself: "Am I solving the right task now?"). The third one is responsible for checking whether one's behaviour corresponds with the implicit system of representations to maintain consistency of the system. We name it "Consistency Control" [1]. We believe that Consistency Control is a higher-level control; it may override lower controls such as Operational and Task control processes. This override happens when one's behaviour greatly deters from expected patterns set by representations. For example, someone has their representation of effectiveness in the game of darts that suggests one has low effectiveness (i.e., they regularly miss the spot where they aimed). They start being highly effective in a particular game scoring many points in one or two sequential throws. However, very often, their next throw is even worse than usual. That happens because a person's Consistency Control found discrepancies between their representation of effectiveness in the game of darts and their actual performance. The Consistency Control finetunes the behaviour to balance out successes with flops. Supposedly, the same is true for the opposite. If one performs poorly in the game, at some point, they may unexpectedly demonstrate one of the best performances. Unfortunately, although the theoretical view presented here cannot be proven directly, we can provide some logically valid inferences that we can test in experiments. Allakhverdov and Allakhverdov [3] review some of those statements and their respective experimental testing. This article investigates another statement on how controls manage predictability and how it influences performance.

No one argues that predictability increases performance. With a few specific conditions (e.g., [6]), people react faster and more accurately to the stimulus they expect. Research suggests that predictability activates task-relevant responses even before the stimulus appears. However, we assume that for that to happen, control mechanisms must evaluate the task as predictable. When this happens, higher-level control mechanisms (that usually require more time to work) decrease their meddling in the works of lower-level mechanisms such as Operational Control. However, researchers face many problems when they want to test this. One of the difficulties is that to induce predictability they use repetition of regularities that participant learns through many trials. First, in most cases, it requires an excessive 
number of trials. By the time the subject learns the regularity, they practice the task long enough for control processes to diminish their influence not only because of predictability but also because the task itself becomes a routine. For example, we may look at the Stroop test (Stroop, 1934), where participants must name the colour of the ink of the word written in an incongruent colour. When the participant faces this task for the first time, they usually perform poorly: they need much more time to name the ink colour of the incongruent colour word compared to the ink colour of a meaningless set of symbols. One of the possible explanations [3] of the interference effect states that the most prominent role in the response delay happens due to Task Control processes. In the Stroop test, participants have two tasks: name the colour of ink and ignore the meaning of a word. The mistakes appear when Task Control inevitably checks whether the cognitive system solves the right task (to name the colour and ignore the word). However, to understand whether the system ignored the meaning of the word, this meaning necessarily appears in consciousness, thus failing to ignore the word. Of course, Task Control receives information that the task is failed. As a result, it increases the response time (This is just a summary of the explanation; for a more detailed description, please see [2]). However, since the Stroop test has many trials and participants are relatively efficient in most of them. After excessive training, the system creates a representation of effectiveness for the Stroop test. When the participant's results closely follow the expected criteria, the performance on the task becomes less controlled, i.e., more automatic. It is evident if we look at participant's performance in the next Stroop test where interference decreases immensely. All of this means that if we create predictable context with regularity, the data may not fully demonstrate the effect of control processing in the Stroop test. We argue that the Musical Stroop Interference Test could be the solution. The Musical Stroop Test is a classic test's modification. In this version, participants respond to incongruent stimuli by naming musical notes placed on the staff and ignoring the written names of another note [4]. The written word could be either inside or outside the note. If it is inside, then most commonly, instead of a notehead, that would represent a position on the staff, the word with the incongruent note name is used, e.g., like the word "la" on the position of "sol". If it is outside the note, the notehead defines the note, while the word with the name of another note is written either to the left, right, above or below the staff.

The interference, in that case, is the difference between average latencies in neutral condition when participants name the notes placed on staff without any distracting information and in the experimental condition when participants respond to musical Stroop stimuli. Previous studies showed the interference effect is the highest when a word substitutes the note compared with when the word is outside. However, our pilot study $(\mathrm{N}=25)$ showed that people demonstrate that musical 
Stroop stimuli cause a noticeable interference effect in trials presenting the incongruent words above and below the staff. The main advantage of using musical Stroop stimuli is that it allows predictable stimuli that do not follow regularities based on repetition but through familiar melodies. Having a melody that participants know makes stimuli predictable. Compared to usual methods, in the Musical Stroop test, predictability appears right away, so it is easier to identify processes connected with experiencing predictability. We argue that after a person recognizes a melody in the target dimension of the Musical Stroop stimuli, their reaction times decrease. To test this hypothesis, we conducted the following experiment.

Twenty-three musical college or conservatory students participated in the study (16 to 25 y.o., 15 females). All of them easily read notes from spreadsheets with violin clef. All participants have never taken any modifications of the Stroop test and have been unfamiliar with the interference effect before the experiment. We carried the study under the Declaration of Helsinki. All participants signed informed consent and participated in the study voluntarily.

We created a specific set of musical Stroop stimuli for our experiment. One part of the stimuli would represent famous, easily recognizable melodies, and the other would be a set of notes in random order. There were nine melodies in the Melody condition and eighteen random sequences in the Filler condition (two fillers for each melody). We used a version of musical Stroop stimuli where names of the notes are above or below the staff to indicate a note value that is essential for melodies. In the filler condition, note values were assigned randomly across a sequence but so that, on average, it had the same note values as in the melody condition. In each trial (one melody or a filler), we used both above and below notations to reduce habituation and make participants noticing them. Finally, we avoided any melody that required using accidentals or rhythmic symbols (rests or dots).

On the one hand, this limitation significantly reduced the range of melodies we could use. On the other hand, it allowed us not to introduce additional unresearched factors (our previous study showed that stimuli' irrelevant characteristics could significantly affect one's reaction). However, we believe that studying the interference effect with notes written with accidentals and rhythmic symbols are valuable for future research. All stimuli were incongruent: a note and the name of the note were always different.

We used the following melodies: 1) "A Morning" by Edward Grieg; 2) Russian folk song "In The Meadow Stood a Little Birch Tree"; 3) a Russian child song "B траве сидел кузнечик" [V trave sidel kuznechik - A Grasshopper Was Sitting in the Grass"]; 4) lullaby song "Twinkle Twinkle Little Star"; 5) a Russian child song "В лесу родилась елочка" ["V Lesu Rodilas' Yolochka" - "In the Forrest, a Christmas Tree is born"]; 6) a Russian child song "Маленькой елочке холодно зимой..." ["Malen'koi 
Yolochke Holodno Zimoi" - "A Small Christmas Tree Is Cold in Winter"]; 7) "Italian Polka" by S. Rachmaninoff; 8) "Песня про зайцев" ["Pesnya pro Zaicev" - "A Song about Hares"] from a movie "Diamond Arm" by Aleksander Zatsepin; 9) A theme from "The Godfather" by Ennio Morricone. All melodies were transcribed, and fillers were created using MuseScore 3.0. The note names above or below staff were added using Photoshop CC. Melodies and fillers had from 12 to 29 notes, totalling up to 623 stimuli in the whole experiment.

Due to the COVID-19 pandemic, we experimented online using the Pavlovia site. Before the beginning of the experiment, we contacted the participant on Zoom, explained the specificities of the experiment, provided an online informed consent form that participants signed by stating their name and pressing "I accept" in the form. Then experimenter sent the link to the experiment and was present throughout the whole procedure. In the beginning, participants had a training stage where they got accustomed to keyboard layout. Each note (Do-Re-Mi-Fa-Sol-La-Si) corresponded to number keys from 1 to 7 in the row (26 stimuli). After participants familiarized themselves with the layout, the main part of the experiment started. Each participant had all 27 trials ( 9 melodies and 18 fillers). Every participant had the same order of the trials: filler 1 - melody 1 - filler 2 - filler 3 - melody 2 - filler 4 etc., until the 18th filler. Each trial started with the appearance of empty staff. Then after 2 seconds, the first stimuli (a note and a word simultaneously) appeared, and the participant had 2 seconds to press a correct number ( 1 for 'Do', 2 for 'Re', etc.). After 2 seconds, the following note appeared and so on until the end of the sequence. The subsequent trial started after 2 seconds of the blank screen. After the completion of all trials, the experimenter asked participants to fill a postexperimental online form. The form consisted of two questions: 1) have they noticed anything during the experiment? 2) what melodies do they know? (They ticked the melodies they know from the list of the nine melodies used in the experiment). After the questions, the experimenter had a debriefing with participants explaining the true aims and procedure of the experiment and answered questions if participants had any. The results of the post-experimental interview showed that most participants (16 out of 23) noticed that some of the sequences were indeed popular and familiar melodies. Ten participants even named the melodies they recognized. The answers to the second question revealed that all songs are mostly familiar to the participants. The most-known melodies were "V trave sidel kuznechik" (22 out of 23 knew the melody), "A Morning" by E. Grieg, "V Lesu Rodilas Yolochka" and "Malen'koi Yolochke Holodno Zimoi" (21 out of 23). The least known melody was a theme from "The Godfather" by E. Morricone (14 out of 23). 17-19 participants knew other melodies. We may say that most people knew the presented melodies, so comparing the melody condition with the filler condition is possible. 
The average time for reacting to stimulus in melodies was $978 \mathrm{~ms}$ (SD $=310$ $\mathrm{ms}$ ), while the average RT for fillers is $1221 \mathrm{~ms}(\mathrm{SD}=376 \mathrm{~ms})$. The difference between two conditions is significant: $t(22)=-12.03, p<.001$. The same is true for accuracy. The participants gave $97.02 \%$ correct answers in the melody condition with only $95.66 \%$ in the filler condition $(t(22)=3.69, p<.001)$. These results show that having predictability in the sequence helped participants react faster to the upcoming stimulus so that the interference effect decreased. All of this corresponds well with previous studies on predictability. However, such a study allowed us to see the role of control processes in this task. To do this, we analyzed each participant's unique patterns of completing each trial. Due to article length, we can provide only one example, and, of course, it is the most exemplary case. However, other participants demonstrated similar behaviour in many of their trials. So let us look at participant \#7. She mentioned in her survey that although she knows a lot of the melodies that were presented to her, there was only one melody she consciously recognized. It was a Russian song, "A Small Christmas Tree is Cold in Winter". Looking at her response times to the melody sequence (Fig. 1A), we see that it is a completely different trend from her reactions to a filler sequence in the previous trial (Fig. 1B).
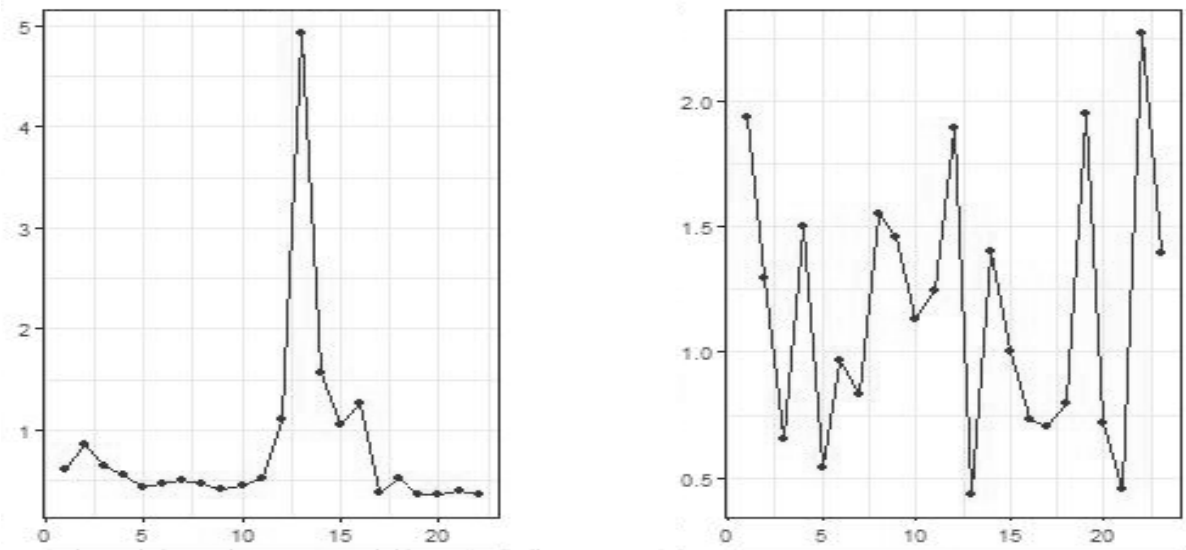

Figure 1. Participant 7's RT plots, in seconds, of A) a melody of Russian song "A Small Christmas Tree is Cold in Winter" on trial \#20 and B) a filler sequence of random notes on trial \#19

We notice that filler has a more sporadic reaction time swinging around one's average time, an expected reaction to a random sequence. Our point of view suggests that the average time approximates one's implicit theory of self-efficiency in this or similar tasks. However, it is noteworthy that it is partially speculation since there is no obvious way to test this hypothesis. A participant sees a note with a word written on top or below the staff, inhibits the incorrect answer and reacts to a note. 
There is no possibility for prediction. Participants try to create a prediction (which is most probably what we always do in case of any systematic and random sequence - we look for regularities), but it almost immediately gets discredited.

In the case of the melody, the situation is the opposite. We also start looking for systematic order in stimuli so that we can predict them. During the sequence presentation on screen, our cognitive system (well before conscious realization) recognizes the song. In the beginning, the response times are sporadic, but soon they become more similar and faster.

At first, there are not enough notes to recognize the melody, hence the randomness of reaction. However, our cognitive systems require just a few notes to find the match - a particular melody. With each subsequent note, it makes a new prediction until suddenly one of the predictions is correct. When this happens, the participant becomes unbelievably (for Consistency Control) successful. So, Consistency Control initiates "investigation": is there a reason for being so effective? Is there any explanation that would allow maintaining an implicit system of representations without any change? The questions do not appear right away but after a few successful performances. When this happens, it results in increased reaction time. In Figure 1A, we see increased latencies from the 12th to 16th note. During that time, we assume Consistency Control interrupts the process. However, as soon as it finds the answer for an unexplainable success, a melody is involved, so the order is predictable and the Operational Control adjusted accordingly, allowing for faster responses. As a result, Consistency Control decreases its influence. Because of that controlling process, the result of the investigation - the name of the melody - reaches consciousness, so the participant is aware that they recognized the song. It gives a person a rational reason to explain one's behaviour and report it in the following survey. After that, the control processes treat this task as automatic with minimal need for monitoring.

We realize that the logic provided here is no more than an ad hoc explanation as of right now. It requires a lot more testing that stumbles into the necessity of a complex experimental design that would be valid for testing the hypothesis without introducing many irrelevant artefacts in the data. We are already planning a set of experiments that will help eliminate some of those unnecessary factors and, hopefully, support our previous findings. Nevertheless, with this said, the results of this study are still significant. First, it shows that using the musical Stroop test is beneficial for studying the specificity of interference effect as a cognitive phenomenon and as a behavioural effect that reveals underlying cognitive control processes. Second, before the experiment, when discussing possible outcomes, we modelled a similar situation. When participants face a melody sequence, we thought they would first demonstrate results similar to a random answering pattern as in the filler condition. However, as soon as the melody is recognized, the response times 
will decrease. In some cases, like in participant \#7's, the acquired data is even more pronounced. Unfortunately, although some of the other data show similar patterns, it is more complex and cannot be interpreted, so additional study is required.

\section{References}

1. Allakhverdov, M.V., Kulieva A.K. A New Look on the Limitations of Cognitive Resources // Individual Abilities and Mental Resources in the World of Global Changes/Способности и ментальные ресурсы человека в мире глобальных перемен, 2020, р. 28-36.

2. Allakhverdov, M. V., Scott, T., Chernaya, A. S., \& Allakhverdov, V. M. Cognitive Control of Irrelevant Stimulus Changes. // Medical Technologies in Medicine/Sovremennye Tehnologii v Medicine, 11(1), 2019, 63-68.

3. Allakhverdov V.M., Allakhverdov M.V. Stroop Effect: Interference as a Logic Paradox // Vestnik of Saint-Petersburg State University. Psychology/Вестник Санкт-Петербургского государственного университета. Психология, 4, 2014, 90-102.

4. Grégoire, L., Perruchet, P., \& Poulin-Charronnat, B. Is the Musical Stroop Effect Able to Keep Its Promises? // Experimental Psychology. 61(1), 2014, pp. 80-83.

5. Kelly, G. Personal construct psychology. Nueva York: Norton, 1955.

6. Korolczuk, I., Burle, B., \& Coull, J. T. The costs and benefits of temporal predictability: impaired inhibition of prepotent responses accompanies increased activation of task-relevant responses // Cognition, 2018, pp. 179, 102-110. 